\title{
The Contribution of Čerenkov Line Radiation to the Broad Lines in AGNs ${ }^{1}$
}

\author{
Hao Wu, Jian-rong Shi, and Jun-han You \\ Institute for Space and Astrophysics, Department of Applied Physics, \\ Shanghai Jiaotong University, Shanghai 200030, China
}

\section{Introduction}

In 1980 , You \& Cheng (1980) suggested that in a dense gas medium, relativistic electrons will produce Cerenkov atomic or molecular emission lines of widths $\Delta \lambda \approx 1-10 \AA$. The Čerenkov line is broader than a normal emission line and has small redshift $\left(\Delta z^{\mathrm{c}} \equiv \Delta \lambda_{p} / \lambda_{l u} \approx 10^{-3}\right)$, so the apparent velocity is about a few hundred $\mathrm{km} \mathrm{s}^{-1}$. We refer to this as the 'Čerenkov redshift'. In 1986, You \& Cheng gave simplified formulae for Čerenkov line emission and the mechanism was confirmed by a series of elegant experiments (Xu et al. 1988, 1989). Recent progress in studies of AGNs, both in theory and observation, provide support for the Čerenkov line-emission model of the BLR of AGNs. In this paper, we prove that for a dense gas, if there are enough relativistic electrons, the Čerenkov line emission is strong enough to compare with the observations, and the Čerenkov line emission dominates over the spontaneous emission lines in the optically thick case.

\section{Contribution to BLR of AGNs}

We accept the conventional photoionization model in which (1) the cloudlets in the BLR are illuminated by a strong UV-X-ray continuum from the central power house (2) an ionized layer is formed at the surface of each cloud (part A), where the emission lines are produced by recombination or collisional excitation. But we emphasize that the cloud in BLR is also illuminated by relativistic electrons (Fig. 1). If the gas is dense enough, Čerenkov line emission is inevitable. The propagation of fast electrons from the center to the BLR is somewhat like a diffusion process, and the distribution of velocities of the fast electrons is isotropic. So there are two line-emission mechanisms that coexist in BLR, namely (1) emission lines produced by spontaneous transitions, and (2) Čerenkov emission lines.

There are two questions have to be answered:

1. Which mechanism is dominant in the BLR?

2. Is the Čerenkov line intensity strong enough to compare with the observed Iobs?

\footnotetext{
${ }^{1}$ This work is supported by National Natural Science Foundation of China.
} 


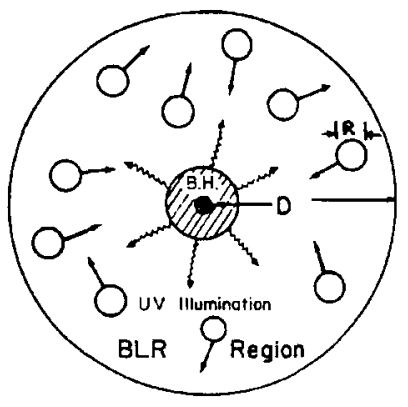

Figure 1. Schematic structure of the BLR.

To answer question (1), we estimate the line emissivity. The spontaneous line emissivity is

$$
J^{s}=\frac{1}{4 \pi} N_{u} A_{u l} h \mu_{l u}
$$

and the Čerenkov hydrogen line emissivity is

$$
J^{c}=1.46 \times 10^{-21} \lambda_{l u}^{2} A_{u l}\left(\frac{g_{u}}{g_{l}}\right) R_{l} N \ln \left(\frac{U_{l i m}}{U_{t}}\right) N_{e} .
$$

Here $N$ is the number density of hydrogen atoms, $g_{u}$ and $g_{l}$ are the degeneracy of the upper and lower levels, respectively, $R_{l}$ denotes the fractional population of the lower level $n=l$ of the hydrogen atom, $N_{e}$ is the number density of relativistic electrons, and $U_{t}$ and $U_{l i m}$ are the Cerenkov line redshift and line width respectively (You \& Cheng 1986).

Under common conditions, we always have $J^{s} \gg J^{c}$. So, for an optically thin gas, we have $I^{c} / I^{s} \simeq J^{c} / J^{s} \ll 1$, so Čerenkov line emission is negligibly small.

But for an optically thick, dense gas, both emission and absorption have to be taken in consideration. As the spontaneous transition line is at the exact position of the intrinsic wavelength $\lambda=\lambda_{l u}$, the emergent spectral intensity $I^{s}$ will be greatly weakened by the large resonance absorption $\left(k_{1}\right)$. But on account of the Cerenkov redshift, the Cerenkov line is located at $\lambda>\lambda_{l u}$, so $I^{c}$ is only affected by the small photoionization absorption $\left(k_{2}\right)$. Therefore the Cerenkov photon can escape from the deep inner part of the cloud, which can make $I^{c} \gg I^{s}$, i.e. a dense gas appears more 'transparent' for Čerenkov lines. In brief, there are two cases have to be considered: Case A, for optically thin gas, where $I^{s} \gg I^{c} \approx 0$, the spontaneous transition lines are dominant; and Case B, in which the gas is optically thick and the relativistic electron density $N_{e}$ is high, we have $I^{c} \gg I^{s}$, and the Cerenkov lines are dominant (Fig. 2). We may argue the possibility of $I^{c} \gg I^{s}$ in another way: if the gas is in thermal equilibrium, the emergent radiation has a blackbody spectrum with no emission lines $\left(I^{s} \approx 0\right)$. However, when relativistic electrons go through the dense gas, the Čerenkov lines will be produced, since this mechanism is a kind of non-thermal line-emission mechanism and it cannot be submerged by the blackbody radiation, and thus 


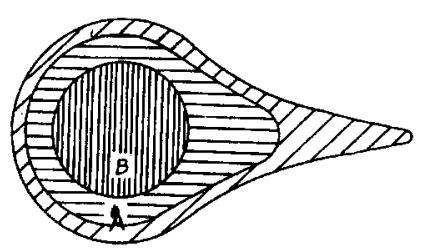

Figure 2. Schematic ionization structure of a small cloudlet.

we have $I^{c} \gg I^{s} \approx 0$. Thus we suggest that for the BLR, the high-ionization lines (e.g., C III], C IV, etc.) are spontaneous transition lines, because they come from the outermost fully ionized layer of a cloud where most hydrogen is the form of $\mathrm{H}^{+}$and therefore corresponds to the optically thin (case A). But the low-ionization and neutral lines (e.g., $\mathrm{Fe}^{+}, \mathrm{Mg}^{+}, \mathrm{H}^{0}$, etc.) are mainly produced by the Cerenkov line mechanism with small 'Cerenkov redshifts' $\left(\lambda>\lambda_{l u}\right)$, as they mainly originate in the inner layer of a cloudlet where the gas is dense, and neutral or partly ionized, and most of the hydrogen is in the form $\mathrm{H}^{0}$. This corresponds to the optically thick case (case B).

To answer the question (2), for example, we estimate the total emergent spectral intensity $I^{c}$ of the Črenkov $\mathrm{H} \beta$ line. For $3 \mathrm{C} 273$, the observed $\mathrm{H} \beta$ luminosity $L^{\text {obs }}(\mathrm{H} \beta) \approx 6 \times 10^{43} \mathrm{ergs} \mathrm{s}^{-1}$. Denoting $\tilde{N}$ the number of cloudlets in BLR of $3 \mathrm{C} 273, R$ the radius of each cloud, $I^{c}(\mathrm{H} \beta)$ the Cerenkov line intensity emerging from the surface of a cloud, and $l^{c}(\mathrm{H} \beta)$ the $\mathrm{H} \beta$ luminosity from each cloud, we have

$$
l^{c}(\mathrm{H} \beta)=4 \pi R^{2}\left(\pi I^{c}(\mathrm{H} \beta)\right)=4 \pi^{2} R^{2} I^{c}(\mathrm{H} \beta) .
$$

The total C̆erenkov $\mathrm{H} \beta$ emission-line luminosity of $3 \mathrm{C} 273$ is thus

$$
L^{c}(\mathrm{H} \beta)=\tilde{N} l^{c}(\mathrm{H} \beta)=4 \pi^{2} \tilde{N} R^{2} I^{c}(\mathrm{H} \beta),
$$

where the unknown parameters $\tilde{N}$ and $R$ can be replaced by the covering factor

$$
C=\frac{\tilde{N} \pi R^{2}}{4 \pi D^{2}}
$$

and the total intensity of the Čerenkov line is

$$
I^{c}=1.46 \times 10^{-19} \lambda_{24}^{-1} A_{42}\left(\frac{g_{4}}{g_{2}}\right) p^{5} \frac{R_{2}}{R_{3}} N_{e} \ln \left(\frac{U_{l i m}}{U_{t}}\right),
$$

where $C \approx 0.1, D$ is the radius of BLR, $R_{2}$ and $R_{3}$ are the fractional population of levels $n=2$ and $n=3$ in the hydrogen atom. The lowest photoionization level is denoted by $p, g_{2}$, and $g_{4}$ are the degeneracy of the levels $n=2$ and $n=4$, respectively, $U_{t}$ and $U_{l i m}$ are the Čerenkov line redshift and line width respectively, and $N_{e}$ is the number density of the relativistic electrons. Taking $R_{2}=10^{-2}$ (due to Ly $\alpha$ trapping), $T=1.0 \times 10^{4} \mathrm{~K}$, and $D=10^{17} \mathrm{~cm}$ (1 light month), we obtain $I^{c}(\mathrm{H} \beta) \approx 0.9 N_{e}$ and $N_{e} \approx 10^{8} \mathrm{~cm}^{-3}$. 
Therefore, $L^{c}$ (or $I^{c}$ ) can be compared with the observed value $L^{\text {obs }}$ (or $I^{o b s}$ ), if the gas is dense and the relativistic electrons are sufficiently abundant. One may ask if the number density of the relativistic electrons $N_{e} \approx 10^{8} \mathrm{~cm}^{-3}$ is too large in the BLR? A possible answer is that this is only a local density of fast electrons near the surface of cloudlet rather than the average density in the whole BLR. According to the model of magnetically confined clouds, the dense gas cloud is confined by the local magnetic field that will also trap the incident fast electrons and cause a local enhancement in the density of relativistic electrons.

\section{Conclusions and Discussions}

1. For a dense gas, Čerenkov emission lines are strong enough to compare with the observations, so the Čerenkov line-emission mechanism is important in BLR.

2. The recombination Ly $\alpha$ line which comes from the outermost fully ionized layer of a cloud is very strong, so the Čerenkov Ly $\alpha$ line emission is negligible. But the $\mathrm{H} \beta$ line is mainly Cerenkov line emission, and thus we can explain the Ly $\alpha / \mathrm{H} \beta$ intensity ratio problem.

3. The different time delays for different lines (Korista et al. 1995) may occur because the low-ionization lines are produced by Čerenkov line emission and the relativistic electrons reach the BLR much more slowly than the photons, due to a random magnetic field in BLR.

\section{References}

Korista, K. T., et al. 1995, ApJS, 97, 285.

Xu, K. Z. 1988, Phys. Rev. A, 33, 2912.

Xu, K. Z. 1989, Phys. Rev. A, 40, 5411.

You, J.H., \& Cheng, F. H. 1980, Acta Phys. Sinica, 29, 927.

You. J. H., \& Cheng, F. H. 1986, Phys. Rev A, 34, 3015. 